\title{
LAS MUJERES Y LA DEPRESIÓN: UNA REFLEXIÓN CRÍTICA
}

\author{
María Mercedes Lafaurie Villamil \\ Universidad El Bosque - Colombia \\ mariamlafaurie@yahoo.com
}

Recibido: 16-02-2010

Aceptado: 04-04-2010

\section{Resumen}

El artículo propone una reflexión en torno al estudio de la depresión en las mujeres desde la perspectiva de la salud mental, partiendo de la crítica feminista y a la luz de algunos hallazgos de la investigación. Describe algunos paradigmas desde donde se explica el por qué los datos epidemiológicos suelen mostrar una incidencia de depresión en las mujeres que duplica aquella hallada en los hombres. Analiza, partiendo de las desigualdades de género, la medicalización del malestar emocional en las mujeres que lleva a que estas constituyan una población con un consumo excesivo de psicofármacos. Palabras clave: mujeres, depresión, género, crítica feminista, medicalización, salud mental.

\begin{abstract}
The article proposes a reflection on the study of depression in women from the view of mental health, on the basis of feminist criticism and under the perspective of some research findings. It describes some paradigms from which it is explained why the epidemiological data often display an incidence of depression in women that duplicates the rate in men. It analyzes, on the basis of gender inequalities, the medicalization of the emotional distress in women, who constitute a population with an excessive consumption of drugs.
\end{abstract}

Key words: women, depression, gender, feminist criticism, medicalization, mental health. 


\section{Introducción}

La depresión constituye un trastorno multifactorial cuya prevalencia es notoriamente mayor en las mujeres, según lo reporta buena parte de la literatura disponible sobre salud mental. La crítica feminista ofrece perspectivas que remueven viejos paradigmas y abren camino a otras formas de valoración e interpretación de esta realidad.

El aporte del feminismo a la inclusión de una perspectiva de género en los asuntos de la salud es innegable y su papel crítico desde la antropología médica, la bioética, la salud sexual y reproductiva, la salud mental y otras disciplinas que profundizan en los aspectos biopolíticos inmersos en el mundo de la salud de las mujeres, es de importancia crucial, atendiendo a que las mujeres enferman y mueren a menudo por causas que son prevenibles y que están asociadas a las desigualdades de género.

Velazco (2006: 11) concibe la teoría feminista como una teoría crítica que, en sincronía con las teorías críticas contextuales, añade una aportación principal, y es que hace aparecer la diferencia sexual como fuente de comprensión de los fenómenos humanos. Se orienta hacia el estudio de las relaciones entre los sexos y sus efectos sobre la vida y salud de cada uno de ellos y se fundamenta sobre el presupuesto de la opresión de las mujeres en el sistema patriarcal, visibilizando una relación de podersubordinación determinada por ese sistema. Para esta autora, "el ser humano tiene sexo y vive y enferma bajo los significados de su sexo (género)”.

Según lo señala Esteban (2006: 11-12) las desigualdades de género en salud representan un campo de investigación en expansión, tanto en las ciencias biomédicas como sociales. La crítica feminista ha tenido una importancia significativa en que esto se dé. La "naturalización" constituye uno de los más importantes cuestionamientos que han surgido a propósito de la lectura que se hace del cuerpo humano en relación con el sexo y otros aspectos biológicos como la raza. Al hablar de naturalización, afirma Esteban, "nos referimos a una percepción absolutamente esencialista y biologicista del cuerpo humano, sobre todo de ciertos cuerpos humanos, que afecta a distintos colectivos (mujeres, negros, homosexuales...), y que lleva implícita su marginación social por 
razones biológicas y anula toda posibilidad de cambio". Añade la autora que entre las aportaciones de la antropología al feminismo ha estado el mostrar las ventajas de tener en cuenta la diversidad cultural y los riesgos de hacer uniforme y universal la realidad de las mujeres; "peligros en los que caen fácilmente algunos discursos con una clara hegemonía dentro del feminismo de la salud, como los que provienen del campo de la medicina y la psicología".

En la perspectiva médica tradicional se acepta y normativiza un rol genérico estereotipado de comportamientos y emociones condicionadas por la cultura que se trata de justificar como "natural" en la mujer, según el planteamiento de Huertas (2009). Para esta autora, tanto a la formación médica como a la psicológica les hace falta tener en cuenta la morbilidad diferencial sexual y genérica y sus causas, tanto respecto a enfermedades exclusivas de las mujeres, o de mayor frecuencia, como en cuanto a la expresión sintomática diversa entre hombres y mujeres en algunas dolencias comunes a ambos sexos.

En este mismo sentido, Ruiz-Cantero y Verdú-Delgado (2004) señalan no sólo los servicios sino la investigación pueden sesgar su práctica, o bien presumiendo erróneamente que la situación de salud de las mujeres y los hombres y sus riesgos son similares o, alternativamente, presumiendo que son diferentes cuando realmente son similares. Según estas autoras las acciones y la investigación derivadas tanto de una como de otra aproximación, menoscaban la salud de ambos sexos, pero más la de las mujeres.

De acuerdo con Valls-Llobet (2006:72-73) en el medio de la salud existe gran desconocimiento de las enfermedades que padecen las mujeres y de cómo prevenirlas. Se requiere, según lo manifiesta esta autora, hablar de "morbilidad femenina diferencial" para "erradicar la cultura del sufrimiento que intenta esconder la ignorancia científica de las causas del malestar y la sintomatología, predicando a las mujeres la resignación y la sumisión a un supuesto destino fatal que las obliga a vivir con molestias y con dolor".

El sistema médico dominante, basado en el conocimiento científico occidental, "ha sido criticado, entre otras cosas por centrarse en los aspectos biológicos teniendo poco en cuenta los sociales, especializándose en un solo órgano o parte del cuerpo, 
olvidándose de la persona afectada" según el análisis propuesto por Tovar (2004: 255) desde la antropología médica. La crítica de la ciencia, evidencia los sesgos de género en la literatura científica, que se asume como objetiva y con validez universal. Por ejemplo, según lo afirma Tovar, existe una tendencia a ver a la mujer como "más frágil", a estar más afectada por tensiones emocionales, a percibir el dolor de diferente manera, e incluso a sufrir de inferioridades, no comprobadas, de orden biológico, asumiendo una distinta composición genética de las mujeres y los hombres.

El creciente reconocimiento de la importancia de las fuerzas externas en la vida de las personas, tales como los factores sociales y económicos, relaciones y ambientes físicos y organizacionales, han conducido a un concepto más amplio de salud mental que el que se conocía hasta hace dos décadas, de acuerdo con lo expuesto por Carmen Torres (n.d).

Plantean Grela y López (2000) que la salud mental de las mujeres representa hoy un campo en revisión y en reconstrucción a la luz de los nuevos modelos conceptuales. La ciencia, los cambios sociales e históricos; la incorporación de las mujeres al mundo del trabajo remunerado y a otras esferas de lo público y las acciones generadas desde los movimientos de mujeres, han puesto en la mirada pública las condiciones de desigualdad en las cuales se ha construido su lugar social.

Nuevas epistemologías, nuevas metodologías y nuevas categorías analíticas han surgido desde el feminismo de cara a la salud mental, especialmente a partir de las décadas de los setenta y ochenta, como reacción frente al determinismo gestor de la llamada "feminización de la locura", según lo expuesto por Ruiz Somavilla y Jiménez Lucena (2003).

La depresión, que constituye la problemática de salud mental más destacada en las mujeres, ha sido motivo de revisión en los últimos años, a la luz de nuevas ópticas. Con el objeto de realizar una aproximación a las miradas, los datos y las explicaciones que se ofrecen en la literatura médica y académica reciente en torno a esta realidad y de proponer una reflexión crítica, se retomará a lo largo de esta aproximación lo que reportan algunos hallazgos sí como se traerán a la discusión valiosos aportes del feminismo a este respecto. 


\section{Las mujeres y la depresión en la literatura científica sobre salud mental}

El género representa hoy en día una dimensión de vital importancia a la hora de analizar realidades de salud y de explicarlas. Dentro de ese contexto, un sinnúmero de informes se refieren a la salud mental como uno de los ámbitos donde se presentan grandes diferencias entre hombres y mujeres, siendo la depresión uno de los aspectos más destacados de estas conclusiones. Las mujeres, según varios informes, se ven afectadas por la depresión en doble e incluso en triple proporción con respecto a los hombres.

De acuerdo con lo expuesto por Vázquez (2005) en los últimos años, y gracias a la visión de género, se ha ampliado la comprensión de los problemas de salud de las mujeres al tiempo que se ha señalado la influencia de su condición de subordinación en las enfermedades que con mayor frecuencia les aquejan. Los problemas de salud mental hacen parte importante de las diferencias de género en la salud, destacándose particularmente la depresión, que se presenta de dos a tres veces más en las mujeres que en los hombres, según lo plantea esta autora. Aspectos como los roles de género, las condiciones socioeconómicas y la autonomía, juegan un papel importante en el estado de salud de las mujeres.

La depresión constituye un trastorno afectivo complejo, definido desde diversidad de abordajes, dependiendo de los contextos teóricos a partir de lo cuales se le describe. Según Zabala-González et al. (2009: 171) la depresión consiste en "episodios leves, moderados o graves de decaimiento del ánimo, con reducción de energía y disminución de la capacidad de sentir placer, el interés y la concentración, con frecuente cansancio, incluso con esfuerzos mínimos". Habitualmente en la depresión el sueño se ve perturbado, se disminuye el apetito y por lo general decae la autoestima y la confianza en si mismo(a), apareciendo a menudo ideas de culpa o de inutilidad. Los problemas depresivos más comunes que han sido referidos y que con frecuencia dan pie a diagnósticos psiquiátricos son, según estos autores: depresión mayor, distimia y desorden bipolar. 
La depresión mayor, suele presentarse mediante episodios depresivos (únicos o recidivantes) que abarcan el pensamiento de las personas, por lo general, la mayor parte del tiempo y pueden implicar ideas recurrentes de muerte y suicidio que no están directamente ligadas a alguna pérdida reciente. En un trastorno distímico los síntomas son considerados más leves o moderados y en el trastorno bipolar se suelen describir variaciones cíclicas de humor. Otras miradas a la depresión son las emanadas de las ciencias sociales, algunas de las cuales asocian esta problemática con la pérdida del sentido de vida y con un conflicto afectivo que toca de manera contundente la esfera existencial de las personas.

Señalan Valls-Llobet et al. (2005) que los últimos estudios sobre las causas de las diferencias de género en cuanto a la depresión apuntan a la necesidad de estudiar el efecto conjunto de las características biológicas y de las experiencias vividas por razón de género. Cada vez se conocen más los efectos de la doble jornada laboral y doméstica sobre la vida emocional. Las mujeres con una posición baja en las escalas de ocupación, que tienen un bajo control sobre el trabajo doméstico y bajo control en el trabajo asalariado se encuentran, según estas investigadoras, en mayor riesgo de sufrir depresión y ansiedad. El cuidado a parientes enfermos, que suele recaer en mayor medida sobre las mujeres, es otro aspecto que es necesario contemplar dentro de aquellos que explican la mayor presencia de depresión en las mujeres, según la visión de estas investigadoras.

Es bien conocida la tendencia a asociar como el origen único y determinante de las depresiones en la mujer el funcionamiento hormonal femenino. Cambios emocionales fisiológicos que en algunos casos pueden ir asociados a unas variaciones hormonales cíclicas específicas de la mujer, son catalogados como síndromes o conjunto de síntomas propios de una enfermedad, según Huertas (2009). Es así como se mencionan el síndrome premenstrual, el síndrome post-parto y el síndrome menopáusico. Algunos estudios han estado centrados en la posible relación entre depresión y estas variaciones fisiológicas, obteniendo resultados que bien vale la pena retomar. 
Ros et al. (2009) llevaron a cabo un estudio epidemiológico con el propósito de conocer la prevalencia de distintos síntomas de depresión y de síntomas físicos y el posible impacto del ciclo menstrual, en una cohorte de 863 mujeres con edades entre 40 y 55 años atendidas en consultas de atención primaria en España. Se trató de un estudio observacional, multicéntrico, transversal, con un solo grupo de pacientes, y con una única visita basal o de inclusión de las pacientes a efectos del estudio, en que se hizo uso de la escala de depresión de Hamilton. Comparando los grupos de pacientes con y sin depresión, los investigadores(as) observaron una mayor proporción de pacientes con síntomas físicos entre las pacientes con depresión que en el grupo sin depresión; por el contrario, comparando según estado menstrual no hallaron diferencias estadísticamente significativas en la presencia de síntomas físicos, en general, entre los grupos de pacientes con menstruación regular, irregular y sin menstruación. Tampoco se hallaron diferencias estadísticamente significativas en la prevalencia de ninguno de los 15 síntomas físicos evaluados excepto en "dolores menstruales" (u otras molestias asociadas a la menstruación), que resultaron más frecuentes entre las pacientes con menstruación irregular, y en "falta de respiración", que resultó más prevalente entre las pacientes sin la menstruación. Este equipo de investigación encontró una alta prevalencia de síntomas somáticos y depresión así como una alta comorbilidad entre ambos por lo que recomiendan la realización de un diagnóstico minucioso de posibles trastornos de depresión y ansiedad que pueden presentar las mujeres de mediana edad, como forma de comprender en mayor medida sus causas y de poner en marcha programas preventivos.

La escolaridad resultó ser un factor determinante para la presencia de depresión en mujeres rurales de 40-59 años que atraviesan la perimenopausia, cuya frecuencia de presentación es indirectamente proporcional al nivel de instrucción escolar, de acuerdo con los hallazgos de un estudio realizado en México por Zabala-González et al. (2009) quienes, además, no encontraron diferencia en cuanto a su frecuencia de presentación en mujeres en la etapa de premenopausia y en aquellas que ya se hallan en la posmenopausia, contrario a otros reportes. 
La depresión postparto ha motivado un sinnúmero de abordajes tanto desde el enfoque biologista como desde el enfoque contextual y desde la perspectiva de la crítica feminista. Manifiestan Valls-Llobet et al. (2005:12) acerca de la depresión postparto, que "diversos factores se han implicado en su patogénesis, pero entre ellos destacan los antecedentes de depresión, un puntaje alto en la escala de acontecimientos vitales y la falta de apoyo social expresada por una mala relación con la pareja”.

Canaval et al. (2000) en un estudio realizado en Colombia con 35 mujeres de estrato socioeconómico bajo hallaron altos niveles de depresión tanto en el preparto $(66 \%)$ como en el post parto(57\%), no encontrándose diferencia significativa para la sintomatología depresiva entre los porcentajes obtenidos en el embarazo y aquellos obtenidos en el postparto. Las autoras recomiendan considerar el apoyo social como posible factor predictor de la depresión experimentada en torno a estas circunstancias por las mujeres con carencias económicas, afectivas y sociales.

La importancia de la experiencia del embarazo, la capacidad para afrontar cambios y el diagnóstico previo de depresión, son reportados como antecedentes de la depresión post-parto por Martínez et al. (2001), a partir de un estudio con 102 mujeres valencianas.

Las madres adolescentes no presentan un riesgo mayor frente a la depresión post-parto que el de otras mujeres, según Latorre et al. (2006) quienes trabajaron en Bucaramanga, Colombia, con una muestra de 286 mujeres de 14 a 43 años.

Fernández-Mateos y Sánchez-Cabaco (2007), establecieron en un estudio realizado con 146 mujeres gestantes en Salamanca, España, que el nivel de estudios de la mujer influye en el estado emocional del proceso de adaptación al embarazo, presentando las mujeres con estudios primarios mayor índice de depresión. La edad, tanto de la mujer como de su pareja, influye en el estado emocional de la mujer gestante, de ahí que, según este estudio, en una edad inferior a 25 años en ambos, se encuentra un mayor índice de depresión. La depresión influye negativamente en la satisfacción marital experimentada y percibida, dificulta la aceptación del embarazo y las relaciones personales e influye negativamente sobre la conducta y salud de la mujer; 
la depresión correlaciona negativamente con el estado de energía y sensación de bienestar experimentados por la mujer gestante y positivamente con estrés.

En Lima, Perú, Luna et al. (2009), realizaron un estudio con el fin de establecer la prevalencia de depresión mayor durante el embarazo. Los autores y autoras de este trabajo establecieron que de las 222 gestantes estudiadas, 89 (40,1\%) presentaron depresión mayor. Las mujeres casadas tendían a deprimirse menos; las que no planificaron su embarazo y que presentaron complicaciones durante su gestación tendieron a deprimirse más que sus pares. No se encontró que la edad, el número de hijos, la edad gestacional, el nivel educacional, los antecedentes de aborto, el consumo de alcohol u otras sustancias, la falta de apoyo familiar y la percepción de tener problemas de pareja, familiares, económicos o personales influyeran en la frecuencia de depresión.

Partiendo de lo anterior, podríamos plantear que aspectos como la cultura, el nivel educativo, las condiciones de vida, las relaciones de pareja y el apoyo emocional y social deben ser considerados al analizar la depresión de las mujeres durante el pre y post parto. Según los estudios reportados, la edad parecería ser un factor de riesgo destacado en España pero no en Colombia, donde la explicación podría estar en que el embarazo juvenil es un fenómeno que está ampliamente extendido y donde las y los jóvenes ven, en principio, la maternidad y paternidad como una oportunidad más que como una amenaza (Lafaurie, 2004). En el Perú, como hemos visto, tampoco resultó significativo este factor al estudiar la depresión mayor durante el embarazo, teniendo mucho más peso el estado civil, la planificación o no del embarazo y las complicaciones durante el mismo.

En un estudio realizado en el contexto de la depresión clínica con mujeres atendidas en Servicios de Psiquiatría de Dubai, Emiratos Árabes, Sualiaman et al. (2003: 30) establecieron, a partir del análisis de los datos sociodemográficos, que existe una significativa diferencia en el nivel de depresión de las pacientes sin alfabetización en comparación con las pacientes alfabetizadas. Las pacientes sin alfabetización tienden a mostrar más síntomas de depresión grave que las pacientes con alfabetización. Esto, según los autores(as), "puede ser debido al hecho de que las pacientes con 
alfabetización disponen de mayores oportunidades para afrontar las dificultades y para expresar sus sentimientos de formas diferentes". Los resultados también indican que las pacientes de clase socioeconómica baja muestran una diferencia significativa mayor en el nivel de depresión que los otros dos grupos, lo cual según este trabajo "puede estar en función de la marginación y estatus social”. Existen límites en el número y en la clase de alternativas de comportamiento al alcance de los individuos que integran la clase baja y esto puede "incrementar una aparente sensación de indefensión e incompetencia”, según explican Sualiaman y su equipo. Este trabajo lleva, nuevamente, a considerar que los aspectos sociológicos así como la marginalidad educativa y social se ven estrechamente relacionados con la depresión en las mujeres. No obstante, es importante resaltar la necesidad de reconocer el peso del contexto cultural al interpretar y valorar las realidades, las expresiones y los comportamientos de las mujeres de las diferentes clases sociales, de cara a la depresión y a su diagnóstico.

\section{Comparación entre mujeres y hombres en estudios sobre la depresión}

La comparación ente mujeres y hombres, en los estudios sobre la depresión, ha ocupado un interés cada vez mayor en la comunidad científica y académica. Tanto la población adolescente como la población adulta han sido objeto de diversos abordajes, buscando avanzar en la comprensión de esta realidad.

Mastern et al. (2003) realizaron un estudio en Sonora, México, no hallando diferencias significativas entre hombres y mujeres adolescentes en la relativo a síntomas depresivos, contradiciendo con ello otros estudios previos.

Más adelante, Balcázar et al. (2007), en un estudio con adolescentes realizado en el Estado de México, encontraron diferencias de género al evaluar la depresión en hombres y mujeres cuando, según sus hallazgos, ellas obtuvieron puntajes significativamente más altos que ellos y "tienden a mostrar más sintomatología depresiva que los hombres". Los autores(as) definen la depresión como un cuadro 
clínico que consiste en un conjunto de manifestaciones de pensamiento y conductas afectivas y somáticas que producen sufrimiento mental. Las diferencias halladas se explican en términos de los diferentes roles sociales ejercidos por los hombres y las mujeres y del hecho de que los varones, según la perspectiva de algunos autores y autoras, cuentan con más mecanismos de afrontamiento frente a la depresión y logran "distraerse hasta que superan su humor", mientras las mujeres "tienden a buscar las razones de su depresión”. La vulnerabilidad cognitiva, creada por Beck, es otra ruta de análisis que tiene en cuenta el equipo responsable de este estudio. Según esta teoría, los trastornos emocionales están asociados a estilos cognitivos los cuales determinan lo que las personas piensan; las relaciones que tienen con los demás y su visión del mundo. Según Balcázar y su equipo es posible que las mujeres presenten "más pensamientos que contribuyen al mantenimiento y desarrollo de la depresión” mientras los hombres presenten ante los mismos hechos más cogniciones relacionadas con conductas externalizantes de carácter violento. De acuerdo con Beck, hombres y mujeres piensan e interpretan las cosas de manera diferente. Sentimientos de rechazo, necesidad de aceptación por parte de los demás, desesperanza, autoculpa y preocupación por las enfermedades, constituyen pensamientos de "autodiálogo negativo" que están presentes en las adolescentes en mayor medida que en los adolescentes varones, según este reporte.

Otro estudio realizado por Saiz et al. (2009) sobre las diferencias entre los géneros exploró las diferencias en cuanto a los síntomas somáticos de la depresión entre varones y mujeres adultos diagnosticados con trastorno depresivo mayor (o depresión severa). La depresión, en la muestra de mujeres, es más frecuente en el grupo de estado civil "casada", seguido de "soltera", "divorciada" y "viuda", mientras que en la muestra de hombres el orden encontrado es "casado", "soltero", "separado" y "viudo". La situación laboral es con mayor frecuencia "activa" tanto en las mujeres como en los varones, seguida por las categorías "ama de casa" o "inactiva", en las mujeres, y "pensionista" o "inactivo" en los varones. Los factores "tratamiento médico" y “acontecimientos vitales en el último mes" resultaron más significativos en la muestra de mujeres. En lo relativo a enfermedades somáticas, éstas se objetivaban en alrededor 
de un $30 \%$ tanto entre ellas como en ellos, sin diferencias significativas. Sin embargo, en las mujeres sí era más frecuente seguir tratamiento médico (40,3\%, frente al 32,5\% en los varones). Los acontecimientos vitales estresantes en el mes previo a la entrevista fueron distintos cualitativamente, siendo sobre todo de tipo familiar en las mujeres (65\%) y de tipo laboral en los hombres (47,5\%), lo que, según explican las investigadoras, parece coincidir con los roles sociales tradicionales para cada género.

No se observaron en este estudio diferencias significativas entre varones y mujeres en la puntuación total en la escala Hamilton de Evaluación de la Depresión (HAM-D17). Los hombres presentaron tasas significativamente mayores en los ítems relativos a las ideas de culpa, alteraciones en la actividad, agitación y síntomas en relación con el aparato genital. Las mujeres presentaron puntuaciones significativamente mayores en los síntomas relacionados con la ansiedad somática y síntomas somáticos generales. Respecto a la frecuencia de síntomas somáticos, los más prevalentes en mujeres fueron dolor de estómago, dolor de espalda, artralgias, cefalea, vértigos, falta de aire, alteraciones gastrointestinales y dispepsia. En los varones, síntomas de la esfera sexual. Estos resultados indican, según el grupo investigador, que a mayor severidad clínica se afecta más el área somática y que a medida que se agrava la esfera clínica también hay mayor deterioro de la calidad de vida del paciente y la paciente.

El principal hallazgo de este trabajo, según sus autoras, fue que las mujeres con trastorno depresivo mayor referían tasas más elevadas de síntomas somáticos que los hombres. Además, esta mayor prevalencia de síntomas somáticos se expresaba también a través de una mayor alteración de la calidad de vida. Otros aspectos psicológicos destacados en las mujeres serían la mayor tasa de expresividad emocional y la amplificación de sensaciones, que podrían desempeñar un papel importante en las características diferenciales de la depresión según el sexo. El rol de género masculino, según las investigadoras, se ha caracterizado como independiente, no emocional y con elevada confianza en sí mismo; por el contrario, el rol femenino sería pasivo, cálido, cuidador, emocional y dependiente. Como la expresión de síntomas depresivos es incompatible con el rol de género masculino, los varones expresarían su depresión de 
forma menos emocional y más aceptada socialmente, es decir, mediante alteraciones en el área social y ocupacional.

El estudio que se acaba de describir, da cuenta de que tanto las mujeres casadas como los hombres casados resultaron ser más vulnerables frente a la depresión, que las personas solteras, separadas y viudas. Sin embargo, un trabajo reciente liderado por Scott et al. (2009) reporta haber hallado a partir del análisis de datos de 34.493 personas de 15 países, que el matrimonio actúa como un factor protector frente a la depresión en ambos sexos, estableciendo entre otras que el matrimonio reduce el riesgo de trastorno por abuso de sustancias en las mujeres más que en los hombres. Este trabajo informa que el divorcio incrementa las posibilidades de depresión en los hombres y el abuso de sustancias en el caso de las mujeres cuando, además, en su primer matrimonio ellas suelen deprimirse en mayor medida que ellos, lo cual explican los autores y autoras a partir de los roles tradicionales que estas suelen ejercer en el hogar.

Vale la pena en este punto traer a colación el estudio realizado por Luna et al. (2009) en el Perú quienes hallaron que el matrimonio es un factor protector frente a la depresión mayor durante el embarazo, estando las mujeres casadas más protegidas que las que no lo son.

La perspectiva desde donde se enfocan los estudios que tienen en consideración el estado civil (o el estatus marital) con respecto a la depresión puede influir sustancialmente en unos y otros resultados obtenidos, además de considerar la importancia que los aspectos culturales y de clase poseen en relación con esta circunstancia. Algunas investigadoras e investigadores recomiendan abordar las relaciones de pareja y su calidad más que el estado civil como tal, atendiendo a que en varios países, especialmente de América Latina, las uniones de hecho son bastante frecuentes así como es importante atender a las múltiples construcciones de familia y de pareja que se observan hoy en la cotidianidad. En algunos de estos países, donde abundan, en las clases más pobres, las mujeres jefas de hogar, el matrimonio suele ser mucho más común en los estratos sociales medios y altos, lo cual es importante tener 
en cuenta al interpretar algunos resultados relacionados con la frecuencia de la depresión en las mujeres y su asociación con el estatus marital.

\section{Depresión y desigualdades entre los géneros: perspectivas de abordaje}

El documento "Desigualdades entre los géneros y salud mental", publicado la OMS (2005) en la Página Web de la ONU, orientará buena parte de este capítulo por su pertinencia y su inmenso aporte al análisis de la depresión en relación con las desigualdades de género. Si bien retoma los datos mundiales relacionados con la depresión en las mujeres, que las consideran doblemente susceptibles frente a la misma, señala este informe que las cifras que le otorgan al sexo femenino un primer lugar en lo relativo a la prevalencia de trastornos depresivos, se deben ver, entre otras, a la luz de su condición de género y de las violencias y desigualdades ante las cuales están expuestas las mujeres de manera cotidiana. A continuación serán revisados algunos de los planteamientos que aparecen en este documento, complementándose la información con estudios que se han realizado dentro de este contexto.

La OMS (2005) destaca la importancia de tener en cuenta que "en el tratamiento de los trastornos psicológicos existen prejuicios por razón de género que los(as) profesionales de la medicina tienen mayor predisposición a diagnosticar depresión a mujeres que a hombres, aunque muestren resultados similares a la hora de medir la depresión o presenten síntomas idénticos y que son altas las probabilidades de que a las mujeres se les receten medicamentos psicotrópicos que alteran el estado de ánimo. Además de los prejuicios relativos a la atención en salud mental, que pueden incidir en el diagnóstico y tratamiento de la depresión en las mujeres, esta organización señala que "también aparecen diferencias de género en los patrones de búsqueda de ayuda para tratar un trastorno psicológico".

Según este organismo, el género determina el poder diferencial y el control que los hombres y las mujeres tienen sobre los determinantes socioeconómicos de sus vidas 
y su salud mental; su posición y su condición social; el modo en que son tratados dentro de la sociedad y su susceptibilidad y exposición a riesgos específicos para la salud mental. Las diferencias de género aparecen en particular en las estadísticas de trastornos mentales comunes (depresión, ansiedad y quejas somáticas). Para esta entidad “estos trastornos, que predominan en las mujeres, afectan aproximadamente a una de cada tres personas en la comunidad y constituyen un serio problema para la salud pública". Señala también la OMS que "la depresión unipolar, que, según las predicciones, será la segunda causa de carga de discapacidad mundial en 2020, es dos veces más común entre las mujeres”.

Plantea este informe que la depresión no sólo es el problema de salud mental más común en las mujeres sino que, además, puede ser más persistente en las mujeres que en los hombres. Destaca igualmente que "los estereotipos de género que indican que las mujeres tienen propensión a los problemas emocionales y los hombres a los problemas con el alcohol parecen reforzar el estigma social y suponen un obstáculo a la hora de solicitar ayuda utilizando las vías normales. Constituyen una barrera para la identificación adecuada y el tratamiento de los trastornos psicológicos".

De acuerdo con Markez et al. (2004:38), se ha demostrado que las mujeres, por razones de índole cultural, están más predispuestas que los varones a reconocer la presencia de enfermedades y a establecer contacto para buscar atención a sus trastornos. "Los diagnósticos de somatización son más frecuentes en las mujeres; la salud se ha psicologizado, pero casi nunca se piensa que las enfermedades de los varones tengan un componente psíquico" afirman estos autores(as) quienes añaden que "estudios recientes indican prevalencias en trastorno mental parecidas en varones y mujeres, aunque las mujeres consultan más". Recordemos el estudio de Saiz et al. (2009) quienes encontraron una mayor adherencia a los tratamientos en las mujeres que en los hombres con depresión mayor.

La violencia relacionada con los problemas de salud mental tampoco se identifica suficientemente, de acuerdo con el análisis de la OMS (2005). Las mujeres son reticentes a revelar que han sido víctimas de violencia a menos que los (as) 
profesionales médicos les pregunten sobre ello directamente. Los trastornos depresivos constituyen cerca del $41,9 \%$ de los casos de discapacidad debida a trastornos neuropsiquiátricos entre las mujeres, mientras que entre los hombres causan el 29,3\%. "La depresión, ansiedad, dolor psicológico, violencia sexual, violencia doméstica y las cada vez mayores tasas de consumo de sustancias afectan en mayor grado a las mujeres que a los hombres en diferentes países y distintas circunstancias”, según afirma la OMS, añadiendo que la presión relacionada con las múltiples funciones que deben desempeñar, la discriminación de género y aquellos factores asociados con la pobreza, el hambre, la malnutrición, el exceso de trabajo, la violencia doméstica y los abusos sexuales "explican la deficiente salud mental de las mujeres".

El vínculo entre las relaciones de pareja y la situación emocional de las mujeres, incluida la depresión, constituye una realidad compleja que resulta ser de primer orden en el campo de la salud sexual y reproductiva. Varios estudios como los descritos por López et al. (2005), Valls-Llobet (2006) y Lafaurie et al. (2005) se refieren a los aspectos psicosociales y emocionales y su impacto sobre la salud reproductiva de la mujeres, siendo la esfera sexual y reproductiva una esfera que se ve tocada ampliamente con la vivencias de situaciones de violencia conyugal y de género.

Eva Espinar (2003) refiere que la depresión hace parte de las consecuencias psicológicas de la violencia contra las mujeres evidenciadas en varios estudios. A este respecto señala Carmen Torres (n.d) que "es claro que la experiencia de haber sido golpeada, violada o maltratada puede llevar a la depresión y ello ha sido demostrado por numerosos grupos de investigación que trabajan con mujeres". Torres afirma que algunos trabajos prueban que las mujeres sometidas a estrés económico y responsables de hijos pequeños presentan más síntomas de depresión. La creciente feminización de la pobreza y las mujeres jefas de hogar, fenómenos ambos a nivel mundial, influyen en la salud mental de las mujeres, de acuerdo con esta autora.

Entre la amplia gama de trastornos de salud mental que padecen las mujeres que han vivido episodios de abuso y, en particular, aquellas que son víctimas de violencia sexual, figuran el estrés postraumático, la depresión, la ansiedad, los 
trastornos del sueño y la alimentación y otros trastornos del comportamiento que afectan la interacción y la sexualidad, según lo señala Calvin Pérez (2001).

Volviendo al informe de la OMS, existe una relación positiva entre la frecuencia y la severidad de los factores sociales asociados a las construcciones de género y la frecuencia y severidad de los problemas de salud mental en las mujeres. Los sucesos graves que causan sentido de pérdida, inferioridad, humillación o aislamiento pueden pronosticar una depresión. La comunicación entre los(as) profesionales de la salud y las pacientes es extremadamente autoritaria en muchos países, lo que implica que el hecho de que una mujer revele sufrir dolor emocional y psicológico sea difícil y a menudo esté estigmatizado. Cuando las mujeres se atreven a contar sus problemas, muchos(as) tienden a tener prejuicios por razón de género que les llevan a dar a las mujeres un tratamiento o excesivo o insuficiente.

Según este organismo los estudios muestran que existen tres principales factores que pueden evitar en gran medida la aparición de problemas mentales, especialmente la depresión: la autonomía; el contar con recursos materiales suficientes y el apoyo psicológico ofrecido por familiares, amigos o profesionales de la salud, que es poderosamente efectivo.

Analizando las diversas aproximaciones a la depresión en las mujeres, a lo largo del trayecto realizado, es posible describir cinco perspectivas desde donde se suelen explicar los datos que reportan una mayor prevalencia de la depresión en las mujeres en comparación con los hombres:

1. Paradigma biologista: enfatiza básicamente en los aspectos hormonales y considera la depresión inherente al sexo femenino.

2. Perspectiva sociocultural: plantea que los hombres por su socialización cuentan con mayores repertorios para afrontar la depresión y para superarla mientras las mujeres se detienen en su conflicto tratando de hallar las razones de su sentir.

3. Teoría de la "vulnerabilidad cognitiva": explica las diferencias en términos de estilos de pensamiento diferentes entre hombres y mujeres, que hacen que 
en las mujeres los pensamientos que conllevan a la depresión sean más frecuentes mientras los hombres ante los mismos hechos suelen pensar de modo diferente y tomar más fácilmente actitudes violentas.

4. Mirada de género: explica, en términos relativos a los roles, relaciones y desigualdades de género el por qué las mujeres se suelen deprimir en mayor medida.

5. Perspectiva crítica feminista: reconoce la existencia de estereotipos de género asociados a las explicaciones relativas a la salud de las mujeres y la predisposición en la medicina occidental a mantener la visión prejuiciada que suele atribuir al sexo femenino problemas de salud mental como inherentes a su condición, presentándose con ello una tendencia a diagnosticar "depresión" en ellas más que en los hombres, así presenten síntomas idénticos, y a otorgarles un tratamiento que o bien sobrepasa las necesidades o que no responde a las mismas.

Las desigualdades de género y las condiciones que rodean las vidas de las mujeres se asocian, según varios de los estudios revisados, a que estas se enfrenten a la depresión en mayor o menor medida. Los sesgos presentes entre profesionales de la salud mental pueden explicar la posible sobrevaloración de la depresión femenina. Esto, merece seguir siendo considerado en futuros trabajos así como es importante no perder de la mira el papel de la violencia de género en el panorama de la depresión de las mujeres.

\section{La medicalización del malestar emocional en las mujeres: los psicofármacos}

Ana Arroba (2003) plantea que el control de nuestros movimientos y actitudes, de nuestras sexualidades y fertilidad, de nuestros ciclos vitales, de nuestros embarazos y partos, de la menopausia, de nuestro envejecimiento, de nuestra salud en su totalidad y de nuestra belleza, está en manos de expertos. El control de nuestros cuerpos es esencial 
para mantener la soberanía patriarcal, y es esencial para mantener la economía en el mundo globalizado. Muchos controlan nuestros cuerpos, y muchos se lucran de nuestros cuerpos, de nuestro sexo, nuestra reproducción, afirma esta autora. Parte de la medicalización es la etiqueta impuesta por el sistema médico a cada mujer diagnosticada y el significado de la enfermedad es inicialmente impuesto por las expectativas culturales sobre tal enfermedad.

Las posibilidades de autonomía que se le plantean a las mujeres se restringen en gran medida debido a dependencia creada en función de las asimetrías económicas y de saber con respecto al personal médico y por un "habitus asistencial" que, en la práctica, no las reconoce como individuas capacitadas para poder decidir más allá del dictamen científico médico, señalan Rostañol y Viera (2003).

Afirma Valls-Llobet (2007: 62) que "en la salud, además de los sesgos en los diagnósticos, también se han producido sesgos de género en los métodos de exploración, en la valoración de la normalidad en los análisis clínicos, y en la aplicación de terapias sin ninguna diferenciación por sexo, ni en dosis ni en vía administrada”.

La mayor prescripción y consumo de fármacos psicótropos en mujeres puede reflejar una mayor prevalencia de depresión y ansiedad en ellas, o que al quejarse induzcan la prescripción más que los hombres. Pero también es posible que en el campo de la medicina se atribuyan los síntomas físicos (o de presentación atípica) a factores psicológicos más fácilmente en las mujeres que en los hombres, o se tienda a prescribir fármacos para síntomas depresivos de baja intensidad a las mujeres más que a los hombres. Lo destacable es que el malestar emocional de las mujeres está medicalizado. Habitualmente, son estrategias terapéuticas sintomáticas equivocadas y "ciegas a las causas”, según lo señalan Ruiz-Cantero y Verdú-Delgado (2004).

Las mujeres somos el grupo principal de consumidoras de psicofármacos, grupo al cual dirigen su publicidad los laboratorios que los producen, afirma Soriano (2006: 17) quien añade que "somos las dos terceras partes de la población que usa tranquilizantes". 
De acuerdo con Valls-Llobet (2008), en el Estado Español el 85\% de los psicofármacos son suministrados a mujeres, lo cual sobrepasa la evidencia de que existe como mucho el doble de ansiedad y depresión en mujeres que en hombres. Señala esta autora que algunas innovaciones en salud, al contrario de mejorar la calidad de vida de las mujeres, incrementan las desigualdades de género.

Partiendo de su análisis, Markez et al. (2004: 56) concluyen que "las mujeres tienen una exposición mayor a la posible prescripción de estos fármacos, ya que los médicos y médicas que estudian la literatura de su especialidad las ven como «más necesitadas» o grupo de riesgo, situación que se une a los estereotipos de género que como personas llevan incorporados en su vida cotidiana".

El principal grupo de riesgo, según Mabel Burin (2004) está constituido por mujeres amas de casa, de mediana edad, de medios urbanos o suburbanos, así como las dedicadas al trabajo maternal con varios hijos pequeños a su cargo, y las que realizan doble jornada de trabajo. Para Burin se trata de mujeres que desempeñan roles de género tradicionalmente femeninos que las sitúan dentro de contextos difíciles de enfrentar. Según esta autora, la problemática se plantea en términos de si es legítima la prescripción excesiva de psicofármacos a las mujeres, o si esto forma parte de una única manera, estereotipada y patriarcal, de diagnosticar y tratar sus conflictos, más bien como forma de control social. Burin propone un mayor apoyo a la investigación y visibilización de este fenómeno. De igual modo, considera que esta situación constituye una prioridad que es necesario reconocer dentro de los programas de salud de las mujeres, mediante estudios y planes de acción destinados a mejorar los criterios de preservación y promoción de la salud. 


\section{A manera de cierre}

Un primer aspecto a destacar, luego del recorrido realizado es que, siendo un constructo complejo, la depresión tiene multiplicidad de formas de ser abordada. Los hallazgos, en ocasiones contradictorios entre unos y otros estudios, llevan a considerar la importancia de reconocer los diferentes paradigmas que contextualizan los abordajes y la necesidad de que los trabajos sobre el tema se enfoquen desde una perspectiva de género, lo que va más allá de comparar datos sobre ambos sexos basándose en visiones cargadas de prejuicios sobre las mujeres y su salud. Es de considerar, igualmente, la relevancia que poseen los aspectos socioculturales y los roles de género en el hecho de que las mujeres experimenten episodios depresivos. La escolaridad ocupa un lugar nada despreciable entre aquellos factores asociados a esta realidad, al juzgar por los hallazgos descritos en varios de los estudios analizados.

Ante la realidad observada, tal cual lo propone Esteban (2006: 13), debemos avanzar en la desnaturalización y desencialización necesaria de los estudios de género, mediante lo cual según la autora "podremos impugnar la misma definición y jerarquización de los síntomas y malestares femeninos que se hace desde la biomedicina y que es asumida acríticamente por las ciencias sociales". Requerimos cuestionar la prioridad de los esquemas "hormonales" y reproductivistas de la fisiología femenina, que según Esteban "determinan de manera radical las ideologías médicas y sociales sobre el ser mujer”. Como lo señala Valls Llobet (2006: 73) es necesario considerar los problemas y las enfermedades específicas de las mujeres, "atendiendo a su queja, su malestar y su dolor".

Se hace necesario el estudio de los padecimientos y del afrontamiento de los mismos desde las perspectivas propias de las mujeres y de los hombres, en sus contextos, constituyendo la investigación cualitativa un recurso valioso frente a esta necesidad. De igual modo, se requiere continuar avanzando en la investigación sobre género y salud mental y en revisiones de la literatura, atendiendo a los estereotipos de 
género, buscando llegar a un diagnóstico diferencial de las enfermedades y del malestar de las mujeres, tal cual lo proponen Huertas (2009) y Valls-Llobet et al. (2005).

Es imperativo trabajar por el empoderamiento de las mujeres con respecto a su salud y por su ejercicio de la autonomía, del auto cuidado y de la responsabilidad personal en este contexto. De igual forma, se requiere fortalecer la inclusión de un enfoque de género en la salud mental y formar al personal de salud en esta perspectiva.

Como lo expresa Leticia Artilés (2008), es necesario desarrollar las fuerzas de la sociedad civil, empoderar a la sociedad, mujeres y hombres, para que adquieran verdadera conciencia de derechos; para que la posición de las mujeres en su acceso al poder, sea real y no formal, y para que en su participación en las políticas públicas dejen de "constituir grupos silenciados" ante el indiscutible dominio patriarcal.

Seguir alimentando el conocimiento que se va adquiriendo sobre la depresión de las mujeres y compartir las reflexiones que surgen desde la crítica feminista, hacen parte de esta importante e impostergable tarea a favor del bienestar de las mujeres.

\section{Agradecimientos}

A María Huertas, psiquiatra y profesora de la UJI-Fundación Isonomía, por haber motivado en mí el interés por profundizar en esta realidad. A Rita Cecilia Plata de Silva, Decana de la Facultad de Enfermería de la Universidad El Bosque, por su juiciosa lectura del manuscrito correspondiente a este trabajo. 


\section{BIBLIOGRAFÍA}

- Arroba A. (2003): "La medicalización del cuerpo en la era de la globalización”. En Página Web de la Asociación Mujeres en Salud, [en línea] Disponible en: http://www.casaames.com/index.php?option=com_content\&task=view\&id=52\&Itemid=80 [Acceso 12/09/2009].

- Artilés L. (2008): "El Nudo Gordiano de la Equidad de Género". En Revista de Salud Pública, 10 Sup. 1, pp. 109-118.

- Balcázar, P. et al. (2007): "Evaluación de la depresión en hombres y mujeres preuniversitarios". En Revista de Neurología, neurocirugía y psiquiatría, vol. 40, nº 4, pp. 97-101.

- Burin, M. (2004): “Ante depresión o stress. La excesiva prescripción de tranquilizantes a mujeres, forma de control social”. En La Jornada, UNAM, 8 de febrero de 2004, [en línea] Disponible

en: http://www.jornada.unam.mx/2004/08/02/informacion/72_medicalizacion.htm [Acceso 20/07/2009].

- Calvin Pérez, M. E. (2001): “Género, Salud Mental y Violencia”. Organización Panamericana de la Salud (OPS), pp.1-8. En Página Web de la OPS (PAHO), [en línea] Disponible en: http://www.paho.org/English/HDP/HDW/mentalhealthandviolence.pdf [21/01/2010].

- Canaval, G. et al. (2000): "Depresión postparto, apoyo social y calidad de vida en mujeres de Cali, Colombia”. En Colombia Médica, nº. 31, pp. 4-10.

- Fernández-Mateos, L. M. y Sánchez-Cabaco, A. (2007): "La depresión en la mujer gestante y su influencia en el proceso de vinculación afectiva prenatal”. En Revista de psicoanálisis, psicoterapia y salud mental, vol. 1, $\mathrm{n}^{\circ}$. 1, [en línea] Disponible en: http://psi.usal.es/rppsm/originales.asp [21/07/2009].

- Espinar, E. (2003): "Violencia de género y procesos de empobrecimiento. Estudio de la violencia contra las mujeres por parte de su pareja o ex-pareja sentimental". Tesis Doctoral, Universidad de Alicante, pp. 1-106. En Página Web Biblioteca Cervantes, [en línea] Disponible en: http://www.cervantesvirtual.com/FichaObra.html?Ref=11683\&ext=pdf\&portal=0 [10/01/2010].

- Esteban, M. L. (2006): "El Estudio de la Salud y el Género: Las Ventajas de un Enfoque Antropológico y Feminista”. En Salud Colectiva, enero-abril, pp.9-20. 
- Huertas, M. (2009): "Impacto de la salud en la vida de las mujeres". Material docente del Master Igualdad y Género en el Ámbito Público y Privado. En UJI-Isonomía, pp. 1-16.

- Grela, C. y López, A. (2000): Mujeres, Salud Mental y Género. Montevideo:Comisión de la Mujer, Intendencia Municipal de Montevideo.

- Lafaurie M. M. (2004): "La cara fecunda de la modernidad". En UnPeriódico, №. 62, agosto 22 de 2004, pp.15, [en línea] Disponible en: http://historico.unperiodico.unal.edu.co/ediciones/62/09.htm [10/08/2009].

- Lafaurie, M. M. et al. (2005): "Women's perspectives on medical abortion in Mexico, Colombia, Ecuador and Peru”. En Reproductive Health Matters, November 2005, Vol. 13, $n^{\circ} .26$, pp. 75-83.

- Latorre, J.; Contreras, L.; García, S. y Arteaga, J. (2006): “La depresión postparto en madres adolescentes de Bucaramanga, Colombia”. En Revista Colombiana de Obstetricia y Ginecología, vol. 57, nº 3, Bogotá Sept. 2006, pp. 156-162.

- López, S.; Gómez P. I. y Arévalo I. (2005): "Violencia contra la mujer. Análisis en el Instituto materno Infantil de Bogotá Colombia, Estudio de corte transversal, 2005”. En Revista Colombiana de Obstetricia y Ginecología, vol. 57, pp. 10-19.

- Luna Matos, M. L.; Salinas Piélago, J. y Luna Figueroa, A. (2009): “Depresión mayor en embarazadas atendidas en el Instituto Nacional Materno Perinatal de Lima, Perú". En Revista Panamericana de Salud Pública, vol. 26, n. 4. Washington, Oct. 2009, pp. 310-314.

- Markez, I. et al. (2004): "Mujeres y Psicofármacos: La investigación en atención primaria". En Rev. Asoc. Esp. Neuropsiq, no. 91, Madrid jul.-sep. 2004, pp. 37-61. [en línea] Disponible en: http://scielo.isciii.es/scielo.php?pid=S021157352004000300004\&script=sci_arttext [12/10/2009].

- Martínez, E. et al. (2001): "La Depresión Postparto: Un Estudio Exploratorio con una Muestra de Mujeres Valencianas”. En Revista de Psicopatología y Psicología Clínica, vol. 6, no. 1, pp. 37- 48 .

- Mastern, W. G. et al. (2003): “Gender differences in depressive symptoms amog Mexican adolescents”. En Anales de Psicología, Vol. 19, n 1(junio) pp. 91-95.

- Organización Mundial de la Salud (OMS), División de Noticias y Medios de Información del Departamento de Información Pública (2005): "Género y salud mental de las mujeres". En Página Web de las Naciones Unidas, [en línea] Disponible en: http://www.un.org/spanish/Depts/dpi/boletin/mujer/genderwomen.html [20/12/2009].

- Ros, S.; Comas, A. y García-García, M. (2009): "Síntomas depresivos y somáticos en mujeres de mediana edad atendidas en Atención primaria". En Semergen, vol. 35, no. 4, pp. 165-172. 
- Rostagnol, S. y Viera, M. (2003): "Derechos sexuales y reproductivos: Condiciones habilitantes y sujetos morales en los servicios de salud. Estudio en el Centro Hospitalario Pereira Rossell, Uruguay, 2003", [en línea] Disponible en: http://74.125.47.132/search?q=cache:fIlCGTcy1 foJ:www.tau.org.ar/upload/ec225b48f3813 b18d3b9eaafbe3a3ed5/Rostagnol_Viera.doc+Arroba+ana+la+medicalizaci\%C3\%B3n+del+ cuerpo+en+la+era+de+la+globalizaci\%C3\%B3n\&cd=2\&hl=es\&ct=clnk\&gl=co [15/12/2009].

- Ruiz-Cantero, M. T. y Verdú-Delgado, M. (2004): "Sesgo de género en el esfuerzo terapéutico". En Gac Sanit, vol. 8 supl. 1, Barcelona, mayo 2004, pp. 32-37, [en línea] Disponible en: http://scielo.isciii.es/scielo.php?pid=S021391112004000400019\&script=sci_arttext [20/07/2009].

- Ruiz Somavilla, M. J. y Jiménez Lucena, I. (2003): “Género, mujeres y psiquiatría: una aproximación crítica”. En Frenia, vol. III-1-2003, [en línea] Disponible en:

http://www.frenia-historiapsiquiatria.com/pdf/fasciculo\%205/007-genero-mujeres-ypsiquiatria-una-aproximacion-critica.pdf [21/11/2009].

- Saiz, D; Rodríguez, M. y García, C. (2009): "Diferencias de sexo en el trastorno depresivo mayor: Síntomas somáticos y calidad de vida”. En Revista de Psiquiatría y Salud Mental. 2009, jul. vol. 2, no. 3, pp. 119-127.

- Scott K. M. et al. (2009): "Gender and the relationship between marital status and first onset of mood, anxiety and substance use disorders". En Psychological Medicine Cambridge University Press, 2009-1-11, [en línea] Disponible en:

http://journals.cambridge.org/action/displayAbstract?fromPage=online\&aid=6699208\&fullt extType=RA\&fileId=S0033291709991942 [20/01/2010].

- Soriano, M. J. (2006): "La Medicalización de la Salud de las Mujeres. La Panacea de los Psicofármacos". En Revista Mujeres y Salud, Invierno 2008-2009, pp. 17, [en línea] Disponible en: http://mys.matriz.net/mys25/img/MYS25.pdf [20/01/2010].

- Sulaiman, S.; Bhugra, D. y De Silva, P. (2003): "Síntomas de la depresión en mujeres de Dubai - Un estudio piloto". En The European Journal of Psychiatry (ed. esp.) v.17 n.1 Zaragoza ene.-mar, pp. 22-32, [en línea] Disponible en: http://scielo.isciii.es/scielo.php?pid=S1579-699X2003000100003\&script=sci_arttext [22/07/2010].

- Torres C. (n.d.): "La otra mirada de la salud mental”. Reflexiones/Salud 4. En Página Web de Isis Internacional, [en línea] Disponible en: http://www.isis.cl/temas/salud/reflex4.htm [16/07/2009]. 
- Tovar, P. (2004): “Una Reflexión Crítica sobre el Género y la Antropología Médica desde la Perspectiva de la Salud y el Cuerpo de la Mujer". En Revista Colombiana de Antropología, $\mathrm{n}^{\circ}$. 40, pp.253-282.

- Valls-Llobet, C. (2006): “La menstruación: de la invisibilidad a la abolición”. En DUODA. Estudis de la Diferencia Sexual. № 31, pp. 71-84, [en línea] Disponible en: http://www.raco.cat/index.php/DUODA/article/viewFile/63189/91493 [24/01/2010].

. (2007): "Salud Comunitaria con perspectiva de género". PACAP. Documentos Comunidad, pp. 61-70. En Página Web de Pacap. [en línea] Disponible en: http://www.pacap.net/es/publicaciones/pdf/comunidad/10/documentos3.pdf [25/01/2010]. . (2008): "La medicalización del cuerpo de las mujeres y la normalización de la inferioridad”. En Innovación para la igualdad, Instituto Vasco de la Mujer, [en línea] Disponible en: http://www.sare-emakunde.com/media2/contenidos/archivos/VallsLlobet.C_08_cast.pdf [24/01/2010].

- Valls-Llobet, C.; Ojuel, J.; López Carrillo, M. y Fuentes, M. (2005): “Manifestación y Medicalización del Malestar en las Mujeres". En Centre d'Anàlisis i Programes Sanitaris, Barcelona, Modulo 4, pp. 1-58.

- Vázquez N. (2005): “Género y Salud”. En Diccionario de Acción Humanitaria y Cooperación al Desarrollo, [en línea] Disponible en: http://dicc.hegoa.efaber.net/listar/mostrar/116 [24/01/2010].

- Velazco, S. (2006): "Evolución de los enfoques de género en salud. Intersección de teorías de la salud y teoría feminista”. Material docente del Diploma de Especialización de Género y Salud Pública. En Escuela Nacional de Sanidad y Observatorio de Salud de la Mujer del Ministerio de Sanidad y Consumo e Instituto de la Mujer del Ministerio de Trabajo y Asuntos Sociales. Madrid, $1^{\text {a }}$ Edición, pp. 1-30.

- Zabala-González, M.; Bautista-Torres, J. y Quebedo-Tejero, E. (2009): “Depresión en mujeres con perimenopausia de una comunidad rural de Huimanguillo, Tabasco, México". En Archivos de Medicina, 2009, vol. 5, nº 4, pp. 171-177, [en línea] Disponible en: http://archivosdemedicina.com/ojs/index.php/archmed/article/viewFile/171/177 [23/01/2010]. 